Research Paper

\title{
Upregulated MMP28 in Hepatocellular Carcinoma Promotes Metastasis via Notch3 Signaling and Predicts Unfavorable Prognosis
}

\author{
Jiangfan Zhou ${ }^{2^{*}}$, Xixi Zheng ${ }^{3^{*}}$, Mei Feng ${ }^{5^{*}}$, Zhichao $\mathrm{Mo}^{2}$, Yunfeng Shan ${ }^{\circledR}{ }^{\bowtie}$, Yilin Wang ${ }^{\llbracket}$, Jing Jin ${ }^{{ }^{\bowtie}}$ \\ 1. Institute of Glycobiological Engineering, Zhejiang Provincial Key Laboratory of Medical Genetics, School of Laboratory Medicine and Life Sciences, \\ Wenzhou Medical University, Wenzhou, Zhejiang, 325035, China. \\ 2. Department of Hepatobiliary Surgery, The First Affiliated Hospital, Wenzhou Medical University, Wenzhou, Zhejiang, 325030, China. \\ 3. Department of Biochemistry and Molecular Biology, School of Basic Medical Sciences, Fudan University, Shanghai, 200032, China. \\ 4. Department of Hepatic Surgery, Fudan University Shanghai Cancer Center; Department of Oncology, Shanghai Medical College, Fudan University, \\ Shanghai, 200032, China. \\ 5. Department of Surgery, Peking University First Hospital, Beijing, 100034, China \\ *These authors contributed equally to this work.
}

$\triangle$ Corresponding authors: Jing Jin, Institute of Glycobiological Engineering, Zhejiang Provincial Key Laboratory of Medical Genetics, School of Laboratory Medicine and Life Sciences, Wenzhou Medical University, Wenzhou, Zhejiang, 325035, China. Tel: +86-577-86689905; E-mail: jinjing@wmu.edu.cn or Yunfeng Shan, Department of Hepatobiliary Surgery, The First Affiliated Hospital, Wenzhou Medical University, Wenzhou, 325000, Zhejiang, PR China. Tel: +86-13857763998; E-mail: shanyf@yahoo.com or Yilin Wang, Department of Oncology, Shanghai Medical College, Fudan University, Shanghai, China. Tel: +86-18117378430. Email: linglingwangyi@126.com.

(c) Ivyspring International Publisher. This is an open access article distributed under the terms of the Creative Commons Attribution (CC BY-NC) license (https://creativecommons.org/licenses/by-nc/4.0/). See http://ivyspring.com/terms for full terms and conditions.

Received: 2018.11.08; Accepted: 2019.01.23; Published: 2019.02.19

\begin{abstract}
MMP28 belongs to the matrix metalloproteinases (MMPs) family and functions in tissue homeostasis and development. Although many other MMPs have been reported to regulate tumor progression, the roles of MMP28 in cancer remain largely elusive. In this study, we investigated the potential roles of MMP28 in hepatocellular carcinoma (HCC). The upregulation of MMP28 was first determined by the analysis on different public datasets. Further quantitative real-time PCR (qPCR) analysis, western blot (WB) assay and immunohistochemistry (IHC) assay on tumor and tumor-adjacent samples from HCC patients confirmed the aberrant elevation of MMP28 in HCC. Pathological analysis showed that increased MMP28 was associated with tumor size, vascular invasion, TNM stage and overall survival in HCC patients. Meanwhile, upregulated MMP28 was identified as an independent prognosis factor in multivariate analysis, and the incorporation of MMP28 expression with TNM staging system established a novel model to improve the accuracy of the predictions. In vivo and in vitro data revealed that MMP28 promoted migration and invasion of HCC cells, and enhanced epithelial-mesenchymal transition (EMT) via elevating zinc finger E-box binding homeobox (ZEB) homologues levels. Furthermore, we determined that Notch3 signaling was critical for the functions of MMP28 in HCC. In conclusion, upregulated MMP28 in HCC promoted migration and invasion and predicted poor prognosis for HCC patients, and the effects of MMP28 depended on Notch3 signaling.
\end{abstract}

Key words: MMP28, Notch3, hepatocellular carcinoma, prognosis

\section{Introduction}

Hepatocellular carcinoma (HCC) is a primary malignant tumor originated from hepatocytes and the most common liver cancer. HCC ranks sixth in neoplasm prevalence and third in cancer-related mortality worldwide [1]. The incidence of HCC in East Asia and sub-Saharan Africa ranks first (over $80 \%$ ), and the number of the patients in China account for $54 \%$ of the world [2]. And the risk of developing HCC in individuals with cirrhosis increases by about $3 \%$ every year [3]. Despite the development of diagnosis and treatment, the mortality of HCC patients remains very high due to the incidence of invasion and metastasis. Meanwhile, although some markers like alpha-fetoprotein (AFP) has been widely 
used in clinic nowadays, their diagnostic accuracy and sensitivity on early and metastatic tumors is still limited[4]. Hence, to cut the cost for cancer survivors and improve their life quality, it is urgent to understand the molecular basis and apply new biomarkers for the precise diagnosis.

In recent decades, the scientific attention on matrix metalloproteinases (MMPs) gains overwhelming growth because of their participation in numerous physiological and pathological processes. MMPs are different zinc-dependent endopeptidases with high heterogeneity [5]. After synthesised as inactive prozymogens, MMPs are activated in the process of extracellular secretion. The activation is regulated by many proteases, such as furins, plasmin, meprin and even other MMPs [6]. Activated MMPs can degrade almost all extracellular matrix (ECM) components, and modulate several signaling pathways via activating or inactivating substrates such as proteases, cytokines, cell-surface receptors [7]. A slight imbalance in MMPs level or MMPs activity leads to pathological conditions for instance vascular diseases [8], autoimmunity disorders [9], and cancer [10]. In cancer, many MMPs are upregulated, which regulates ECM remodeling thus to promote tumor invasion, and eventually promote tumor progression [11]. Among these MMPs, MMP2, MMP9, and MMP13 are overexpressed in HCC, and have been used for HCC diagnosis [12]. MMP28, also named epilysin, is the newest identified MMP [13]. Like other MMPs, MMP28 is cleaved in the Golgi apparatus and released as an active hydrolase. MMP28 have been detected in some normal tissues [14], closely associated with cell proliferation during epithelial repair [15]. The aberrant upregulation of MM28 has been detected in different cancers, including gastric carcinoma [16], grade I squamous cell carcinoma (SCC)[15], and invasive ductal cell carcinoma[17]. However, the expression and functions of MMP28 in HCC remain unclear.

Notch signaling pathway is crucial for tissue development, and is transduced by the transmembrane ligands of the Jagged (Jagged1 and 2) and Delta-like (DLL1, 3, and 4) proteins expressed on the neighboring cells [18]. Four Notch receptors, known as Notch1, 2, 3 and 4, have been identified, and all of them have 3 domains including an extracellular fragment, a transmembrane subunit and an intracellular domain. Ligand binding to the extracellular segment triggers cleavage events on the Notch intracellular domain (NICD), and then NICD is released into the cytoplasm [19]. Afterwards, NICD translocates into the nucleus and binds to the transcription factor CBF1/RBP-Jk to form the transcriptional activation complex that ultimately activates the target genes [20]. Notch receptors and their ligands have been detected in the human mature liver, and the dysregulation on their expression is closely associated with liver diseases including HCC [21]. However, although perturbed Notch signaling or aberrant expression of Notch proteins have been observed in HCC, their effects on HCC progression remain controversial. For example, although Notch1 is upregulated in HCC [22], and Notch1 signaling promotes the hepatocarcinogenesis in animal models [23], Notch1 was reported to suppress HCC in some cases [24], and overexpressing active Notch1 triggers cell cycle arrest and apoptosis [25]. Similar controversial effects have been observed in Notch3 $[26,27]$ and its abnormal accumulation in HCC [28]. In the present study, we determined that MMP28 functions via Notch3 signaling in HCC, and analyzed the potential mechanism how MMP28 and Notch3 regulates HCC progression.

\section{Materials and Methods}

\section{Patients and specimens}

A total of 117 pairs of tumor and adjacent normal tissue specimens were enrolled in the present study, and these patients had undergone hepatectomy from February 2010 to May 2011 or from December 2014 to August 2015, at Department of General Surgery, First Affiliated Hospital of Wenzhou Medical University (Zhejiang, China). All participants were selected if they were pathologically diagnosed with HCC. The adjacent normal tissue specimens were taken at a distance of $3 \mathrm{~cm}$ from the tumor tissues during surgery. Among them, 30 pairs of surgical sections were cryopreserved at $-80^{\circ} \mathrm{C}$ for western blot analysis, and another 87 pairs were formalin-fixed and paraffin embedded for Immunohistochemistry analysis. Each pair of specimens has its own hospital number, the clinicopathological characteristics of the patients including age, gender, tumor size, lymph node invasion, distant metastasis, and tumor stage were recorded in detail. Tumor stages were histologically classified according to the 7th Edition of the American Joint Committee on Cancer TNM classification. All methods were approved by the research medical ethics committee of Wenzhou Medical University (Zhejiang, China) and were carried out in accordance with the approved guidelines. The written consent conforming to the ethical guidelines of the Helsinki Declaration were obtained from all patients before the start of the experiment.

\section{Animal study}

Five to six-week-old male Balb/c nude mice were purchased from Shanghai Laboratory Animal Center of Chinese Academy Sciences (Shanghai, 
China) and were housed in a separate pathogen-free room. All of the animal experiments were approved by the research medical ethics committee of Wenzhou Medical University and carried out in accordance with the approved guidelines. All of the mice were randomly grouped ( $n=6$ in each group). For the lung metastasis model, Huh7-luc cells transfected with MMP28 expression vector were resuspended in ice-cold PBS $\left(1 \times 10^{6}\right.$ cells/mouse in $100 \mu \mathrm{l}$ PBS $)$ and were injected into mice through the tail vein. Four weeks later, all the mice were monitored for bioluminescence every week with IVIS200 (Xenogen, Caliper, CA) after intraperitoneal administration of $200 \mu \mathrm{l}$ luciferin (at $15 \mathrm{mg} / \mathrm{ml}$; Promega, WI, USA). The luciferase signals intensity was calculated by ROI analysis.

\section{Western blot analysis}

HCC tissues were homogenized with RIPA buffer (Beyotime, Institute of Biotechnology, Jiangsu, China). After boiled, samples were loaded in SDS-polyacrylamide gel electrophoresis, transferred to polyvinylidene difluoride membranes (PVDF; Millipore, Bedford, MA, USA), and incubated with primary antibodies. Antibodies against MMP28, Notch 3 and GAPDH were used. The membranes were further incubated with IgG-HRP secondary antibodies (Santa Cruz Biotechnology) and visualized using an enhanced chemiluminescence kit (Tiangen Biotech, Beijing, China) in the image analyzer ImageQuant LAS 4000 (GE Healthcare, Abingdon, UK).

\section{Quantitative real-time PCR}

Total RNA was extracted from HCC tissues using TRIzol (Ambion and life technologies, CA, USA) according to the manufacturer's instructions. Reversed-transcriptional PCR (RT-PCR) was performed using a PrimeScript ${ }^{\mathrm{TM}} \mathrm{RT}$ reagent Kit (TaKaRa, Dalian, China), and the resulting cDNA was analyzed by quantitative real-time PCR using SYBR® Premix Ex Taq $^{\mathrm{TM}}$ (TaKaRa). GAPDH was controlled as a reference gene. The primers used were as follows:

Table A. Primer Sequences

\begin{tabular}{ll}
\hline Gene & Primer Sequence \\
\hline MMP28 & $\begin{array}{l}\text { forward, TCCCACCTCCACTCGATTCAG } \\
\text { reverse, GCCGCATAACTGTTGGTATCT } \\
\text { ZEB1 }\end{array}$ \\
forward, GATGATGAATGCGAGTCAGATGC \\
reverse, ACAGCAGTGTCTTGTTGTTG \\
forward, CAAGAGGCGCAAACAAGCC \\
reverse, GGTTGGCAATACCGTCATCC \\
fnail & $\begin{array}{l}\text { forward, TCGGAAGCCTAACTACAGCGA } \\
\text { forward, AGATGAGCATTGGCAGCGAG }\end{array}$ \\
E-cadherin & $\begin{array}{l}\text { reverse, GGGTGTCGAGGGAAAAATAGG } \\
\text { forward, TCAGGCGTCTGTAGAGGCTT } \\
\text { N-cadherin }\end{array}$ \\
reverse, ATGCACATCCTTCGATAAGACTG \\
forward, GACGCCATCAACACCGAGTT \\
reverse, CTTTGTCGTTGGTTAGCTGGT
\end{tabular}

\begin{tabular}{|c|c|}
\hline$\beta$-catenin & $\begin{array}{l}\text { forward, AAAGCGGCTGTTAGTCACTGG } \\
\text { reverse, CGAGTCATTGCATACTGTCCAT }\end{array}$ \\
\hline Notch3 & $\begin{array}{l}\text { forward, TGGCGACCTCACTTACGACT } \\
\text { reverse, CACTGGCAGTTATAGGTGTTGAC }\end{array}$ \\
\hline Notch4 & $\begin{array}{l}\text { forward, TGTGAACGTGATGTCAACGAG } \\
\text { reverse, ACAGTCTGGGCCTATGAAACC }\end{array}$ \\
\hline Akt & $\begin{array}{l}\text { forward, AGCGACGTGGCTATTGTGAAG } \\
\text { reverse, GCCATCATTCTTGAGGAGGAAGT }\end{array}$ \\
\hline PKG-I & $\begin{array}{l}\text { forward, CTTGGAGCTGTCGCAGATCC } \\
\text { reverse, TCTTTGATGATGCAACTGTCCTT }\end{array}$ \\
\hline VASP & $\begin{array}{l}\text { forward, ATGGCAACAAGCGATGGCT } \\
\text { reverse, CGATGGCACAGTTGATGACCA }\end{array}$ \\
\hline GAPDH & $\begin{array}{l}\text { forward, GAGTCAACGGATTTGGTCGT } \\
\text { reverse, TTGATTTTGGAGGGATCTCG }\end{array}$ \\
\hline
\end{tabular}

\section{Tissue microarray and Immunohistochemistry}

Tissue microarrays (TMAs) were constructed from surgical specimens that are formalin-fixed and paraffin embedded. Chips of $1 \mathrm{~mm}$ diameter were punched from cancer tissues and paracarcinoma tissues respectively. Immunohistochemistry analysis was applied on tissue microarray using Dako REAL EnVision Detection System (Dako, Denmark) according to the manufacture instruction. Primary antibodies against MMP28, ZEB-1, ZEB-2, E-cadherin, $\mathrm{N}$-cadherin, and Notch3 were used to quantify the relative expression level. Hematoxylin was used for counterstaining. The staining intensity was scored as 0 for negative; 1 for weak; 2 for moderate weak; 3 for moderate strong and 4 for strong. The score for the stained area was set as 1 for $0 \%-33 \% ; 2,33 \%-66 \%$; and $3,66 \%-100 \%$. The final staining score was obtained by multiplying staining intensity score with staining area score, and the results are a series number ranging from 0 to 12 .

\section{Cell lines and reagents}

All the human HCC cell lines including BEL-7402 and Huh7 were obtained from Cell Bank of Type Culture Collection of Chinese Academy of Sciences (Shanghai, China). Cells are cultured in Dulbecco's modified Eagle medium (Sigma-Aldrich, St Louis, MO, USA) replenished with $10 \%$ fetal bovine serum (Gibco, Grand Island, NY, USA) at $37^{\circ} \mathrm{C}$ in a humidified incubator containing 5\% CO2. Antibodies against MMP28, Notch3, Flag-tag and GAPDH were purchased from Proteintech (Chicago, IN, USA). Antibodies against ZEB-1, ZEB-2, Snail, E-cadherin, $\mathrm{N}$-cadherin, Vimentin, $\beta$-catenin, Notch4, Akt, phospho-Akt (Ser473/Thr308), PKG-I, VASP and phospho-VASP (Ser239) were purchased from Cell Signaling Technology (Beverly, MA, USA).

\section{Plasmid construction, siRNA and transfection}

The cDNA encoding human MMP28 was obtained by PCR and cloned into the pCMV-Flag vector (Sigma, St. Louis, MO, USA) to generate pCMV-Flag-MMP28 construct. The siRNA targeting 
MMP28 was purchased from Proteintech (Chicago, IN, USA). Transient transfections were performed with Lipofectamine 3000 (Invitrogen) according to the manufacturer's instructions.

\section{CRISPR/Cas9 system construction}

The sgRNAs were designed on E-CRISPR to disrupt the target DNA region of Notch3, and the reverse sequences were completed. The sequence for sgRNA was GGGTGAGCGGTGTCAGCTGG. The oligos was flanked on the 3 ' end referred to the target site sequence (20bp). After annealing, DNA oligos was inserted into PX459 plasmid vector according to the manufacture instruction. Stable cell lines were generated with puromycin $(1 \mu \mathrm{g} / \mathrm{ml})$ in the medium.

\section{Transwell assay}

Transwell migration and invasion assays were carried out with 12-well transwell plates containing 8- $\mu \mathrm{m}$ pore filters (Corning, New York, NY, USA). For invasion assays, the bottom of transwell chamber was coated with BD Matrigel Basement Membrane Matrix (BD Biosciences, San Diego, CA, USA). $1 \times 10^{5}$ cells were seeded into the upper chamber in $500 \mu l$ serum-free basic medium, and the lower chamber was filled with $1500 \mu \mathrm{l}$ complete culture medium. The migration and invasion systems were incubated for 24 $\mathrm{h}$ and $48 \mathrm{~h}$ at $37^{\circ} \mathrm{C}$ respectively. Cells on the upper side of the chambers were removed by scrubbing, and cells on the lower surface of the membrane were fixed with $4 \%$ paraformaldehyde for 15 minutes. Then it was stained with $0.1 \%$ crystal violet (Beyotime Institute of Biotechnology, Jiangsu, China) for 30 minutes. The infiltrating cells were viewed and counted in five randomly selected fields under a microscope.

\section{GEO and TCGA datasets}

Data involved in this study are available from the Cancer Genome Atlas (TCGA) and the GEO datasets (accession number: GSE36376, GSE25097, GSE39791). The number of tumor specimens in each sub-database is equal to the number of paracancerous specimens. For TCGA dataset, mRNA levels were measured by RNA sequencing V2 RSEM. For GEO dataset, mRNA levels were achieved from Oncomine database.

\section{Statistical analysis}

IBM SPSS Statistics 22.0 (SPSS Inc, Chicago, IL), Prism 6.01 software (GraphPad, La Jolla, CA) and R software 3.4.1 with the "rms" package (R Foundation for Statistical Computing, Vienna, Austria) were used for all data analyses. ROC curves were conducted to determine the cut-off value of the Immunohistochemistry staining score to divide the specimens into two groups and compare the prognostic sensitive and specificity of combination model. To evaluate the correlations the between MMP28 expression and clinicopathologic variables, independent sample $t$-test was used to analyze continuous variables, and chi-squared test was applied for categorical variables. Kaplan-Meier method was used to establish survival curves and the significance was calculated by Log-rank test The Cox proportional hazard regression analysis was employed to perform multivariate analyses. Nomogram was displayed by $\mathrm{R}$ software with the "rms" package and the C-index and AIC were calculated to measure the prognostic accuracy. The gray value of the western blot band was quantified by ImageJ 1.8.0 (National Institutes of Health, USA). Differences between groups were determined with Student's $t$ test. Statistical significance was set at two-tails $P<0.05$.

\section{Results}

\section{MMP28 is overexpressed in Hepatocellular Carcinoma}

To determine whether MMP28 is involved in HCC progression, we first examined its mRNA levels in different public datasets from Gene Expression Omnibus (GEO) and The Cancer Genome Atlas (TCGA) database. Data revealed that MMP28 levels were significantly increased in tumor tissues in GSE36376 [29] $(P<0.001)$, GSE25097 [30] $(P<0.001)$, GSE39791 [31] $(P<0.001)$, and TCGA [32] datasets $(P$ $=0.007$ ) (Fig. 1a). To verify the upregulation of MMP28 in HCC, we next examined MMP28 levels in 30 paired HCC tissues and adjacent normal tissues. Both the western blot and quantitative real-time PCR (qPCR) analysis revealed that $66.7 \%(20 / 30)$ of primary tumor tissues expressed more MMP28 compared with matched paracancerous tissues, and statistical analysis verified that MMP28 was significantly upregulated in both mRNA and protein levels $(P<0.001$ in western blot and $P=0.037$ in qPCR analysis) (Fig. 1b-d). We further applied immunohistochemistry (IHC) assay on a tissue microarray including other 87 pairs of HCC samples, and confirmed the significant upregulation of MMP28 in HCC tumor tissues $(P<0.001)$ (Fig. 1e, f). Our IHC results also revealed that MMP28 was mainly expressed in cytoplasm and extracellular matrix (Fig. $1 e)$.

\section{Correlations between MMP28 expression and clinicopathologic characteristics of HCC patients}

To explore the clinicopathologic significance of MMP28 in HCC, we further analyzed the IHC data. 
The receiver operating characteristic (ROC) curve was established and the patients were eventually divided into two groups according to the cut-off value of IHC score 6. Among 87 cancer specimens, 53 (60.9\%) conferred high expression of MMP28. The representative IHC staining was showed (Fig. 2a). The correlations between MMP28 expression and clinicopathologic characteristics were analyzed by chi-square test

a
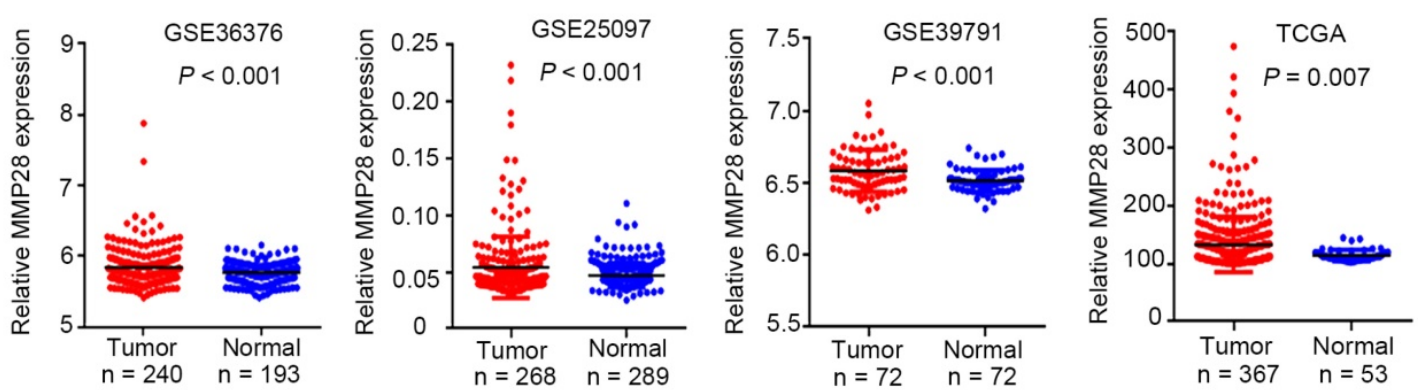

b
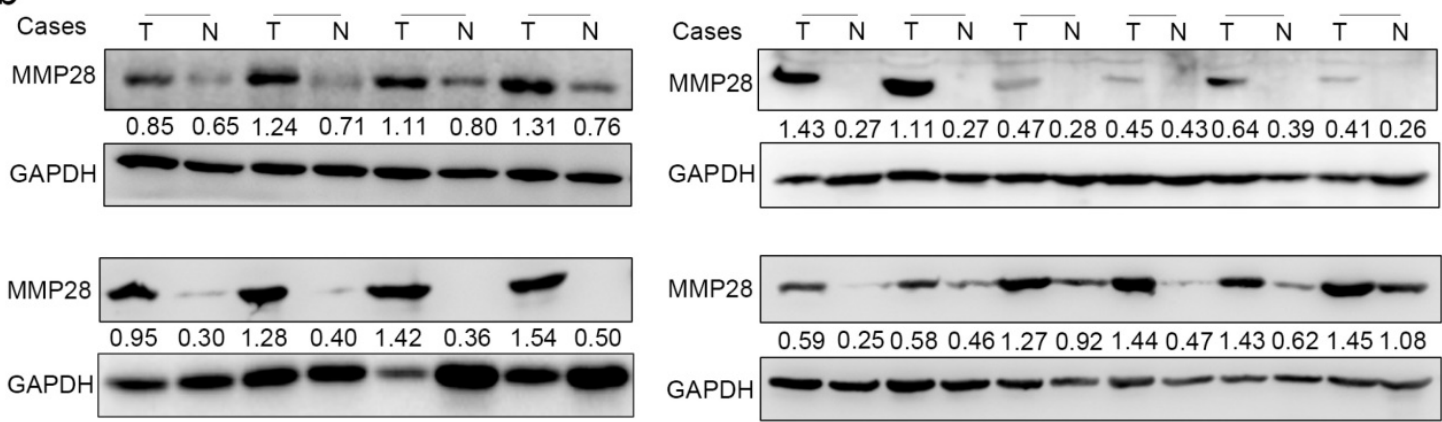

C

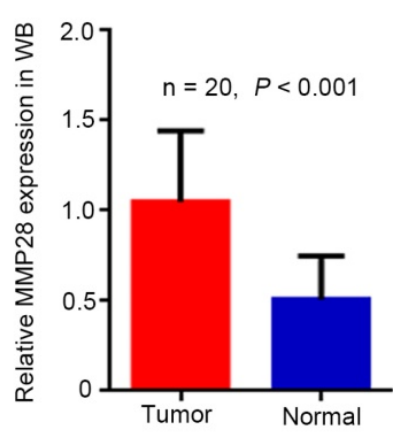

e
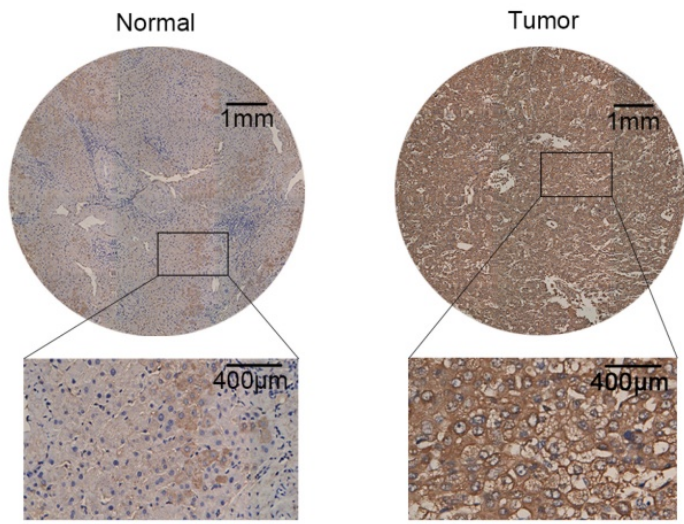

d

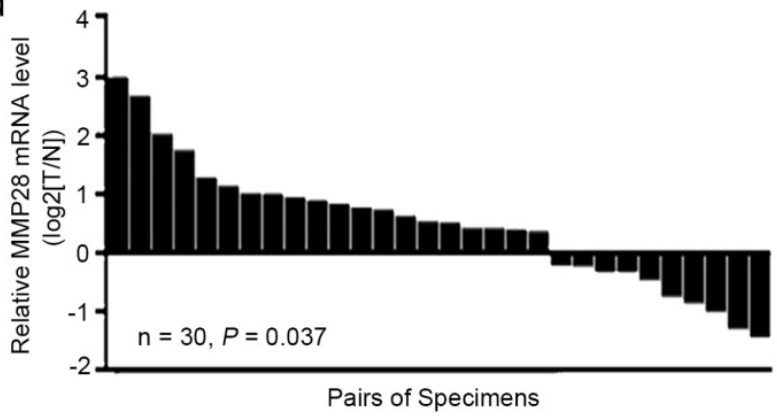

f

Figure 1. MMP28 was upregulated in hepatocellular carcinoma. (a) Relative expression of MMP28 mRNA in HCC tissues and normal paracancerous tissues in GSE36376, GSE25097, GSE39791, TCGA datasets. (b-d) The expression of MMP28 was detected by western blot (b, c) and real-time PCR (d). (e) Representative IHC images of MMP28 protein staining in tissue sections. Regional magnification images were shown below. (f) The score of MMP28 expression in 87 paired HCC tissue sections determined by IHC assay. 
a

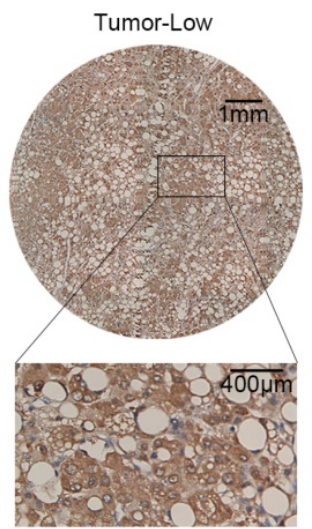

b
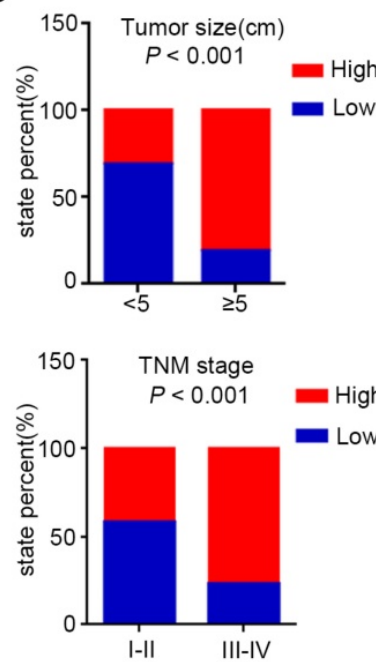

e

MMP28 expression(High vs Low)

TNM(III-IV vs I-II)

Vascular invasion(Positive vs Negative)

Tumor size $(\geq 5 \mathrm{~cm}$ vs $<5 \mathrm{~cm})$

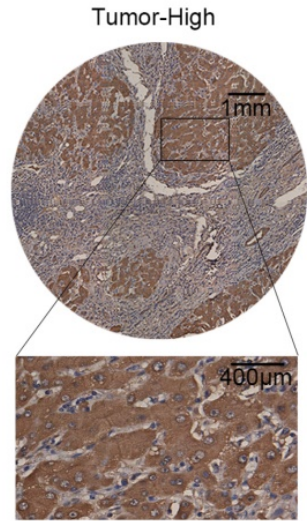

C

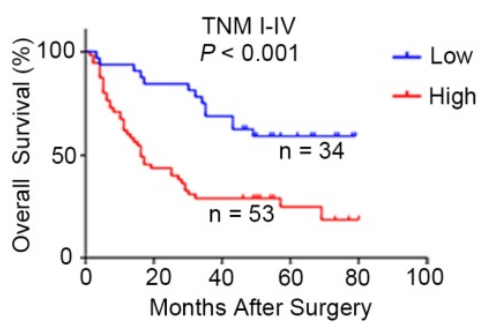

d
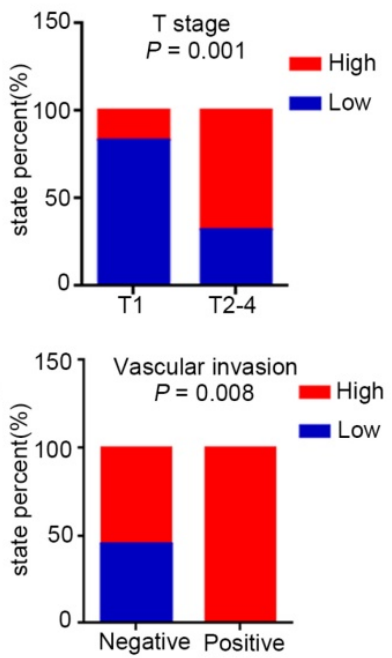

Multivariate Cox regression analysis

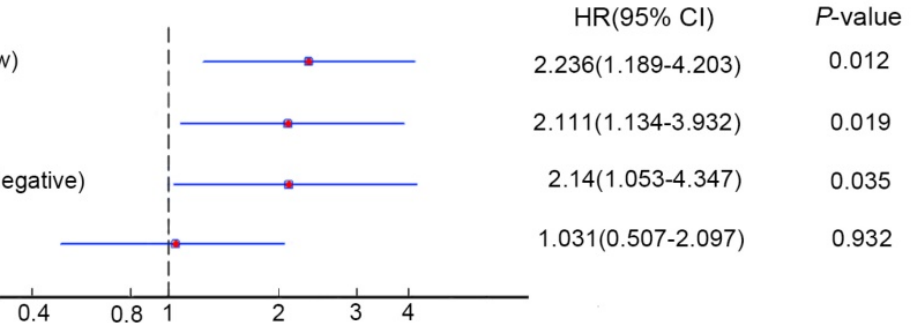

Figure 2. MMP28 was correlated with the poor prognosis of HCC patient in IHC cohort. (a) Low and high expression of MMP28 protein in HCC tissue sections determined by IHC. Representative images were shown. (b) The correlations between MMP28 expression and the clinicopathological variables in IHC cohort. (c, d) Kaplan-Meier survival curves for the overall survival of the delaminated HCC patients from IHC cohort. (e) Multivariate Cox regression analysis showing the independent prognostic factors for overall survival.

We next used Kaplan-Meier analysis to evaluate the relationship between MMP28 levels and the overall survival (OS) of HCC patients. The results indicated that overexpressed MMP28 was significantly associated with poorer overall survival $(P<0.001)$ (Fig. 2c). To further explore whether MMP28 expression could be a stratifying factor in HCC patients within different TNM stages, we dichotomized these subjects into two groups: early stage (TNM I-II) and advanced stage (TNM III-IV). The survivorship curves showed significant value to predict the prognosis of HCC patient in both early and advanced stages (Fig. $2 \mathrm{~d})(P=0.035$ in early stage and $P=0.024$ in advanced stage).

\section{MMP28 can be used as an independent prognosis factor in patients with HCC}

We also used univariate analysis to evaluate the prognostic factors for overall survival in HCC patients. TNM stage (HR, 2.871; 95\% CI, 1.648-5.003; $P$ $<0.001)$, MMP28 expression (HR, 2.778; 95\% CI, 1.505-5.129; $P<0.001)$, tumor size (HR, 1.992; 95\% CI, 1.133-3.503; $P=0.017$ ), and vascular invasion (HR, 3.577; 95\% CI, 1.866-6.855; $P<0.001)$ were identified 
as significant risk factors that could affect the survival in patients with HCC (Table. 2). Further multivariate Cox analysis revealed some independent predict factors for overall survival of HCC patients including MMP28 (HR, 2.236; 95\% CI, 1.189-4.203; $P=0.012$ ), TNM stage (HR, 2.111; 95\% CI, 1.134-3.932; $P=0.019)$ and vascular invasion (HR, 2.14; 95\% CI, 1.053-4.347; $P=0.035)$ (Fig. 2e). These results indicated that MMP28 could be used as an independent prognostic variable in HCC patients.

Table 1. Correlations between MMP28 expression and clinicopathological variables of HCC patients

\begin{tabular}{|c|c|c|c|c|}
\hline \multirow[t]{2}{*}{ Variables } & \multirow[t]{2}{*}{ Number } & \multicolumn{2}{|c|}{ MMP28 expression } & \multirow[t]{2}{*}{$P$-value } \\
\hline & & Low (\%) & High (\%) & \\
\hline Gender & & & & 0.054 \\
\hline Male & 53 & $25(28.7)$ & $28(32.2)$ & \\
\hline Female & 34 & $9(10.3)$ & $25(28.7)$ & \\
\hline Age (years) & & & & 0.426 \\
\hline$<60$ & 63 & $23(26.4)$ & $40(46.0)$ & \\
\hline$\geq 60$ & 24 & $11(12.6)$ & $13(14.9)$ & \\
\hline Tumor size $(\mathrm{cm})$ & & & & $<0.001$ \\
\hline$<5$ & 35 & $24(27.6)$ & $11(12.6)$ & \\
\hline$\geq 5$ & 52 & $10(11.5)$ & $42(48.3)$ & \\
\hline Tumor number & & & & 0.915 \\
\hline Single & 48 & $19(21.8)$ & $29(33.3)$ & \\
\hline Multiple & 39 & $15(17.2)$ & $24(27.6)$ & \\
\hline Pathological stage & & & & 0.12 \\
\hline I-II & 58 & $26(29.9)$ & $32(36.8)$ & \\
\hline III-IV & 29 & $8(9.2)$ & $21(24.1)$ & \\
\hline T stage & & & & 0.001 \\
\hline $\mathrm{T} 1$ & 12 & $10(11.5)$ & $2(2.3)$ & \\
\hline $\mathrm{T} 2-\mathrm{T} 4$ & 75 & $24(27.6)$ & $51(58.6)$ & \\
\hline TNM stage & & & & $<0.001$ \\
\hline I-II & 40 & $23(26.4)$ & $17(19.5)$ & \\
\hline III-IV & 47 & $11(12.6)$ & $36(41.4)$ & \\
\hline Vascular invasion & & & & 0.008 \\
\hline Negative & 75 & $34(39.1)$ & $41(47.1)$ & \\
\hline Positive & 12 & $0(0)$ & $12(13.8)$ & \\
\hline
\end{tabular}

Abbreviations: $P<0.05$ is considered to have statistical significance.

Table 2. Univariate Cox regression analysis of clinicopathological characteristics influencing the overall survival of HCC patients

\begin{tabular}{lccc}
\hline Variables & \multicolumn{2}{l}{ Univariate } & \\
\cline { 2 - 4 } & HR & $\mathbf{9 5} \% \mathbf{C I}$ & $P$-value \\
\hline $\begin{array}{l}\text { Gender } \\
\text { Male vs Female }\end{array}$ & 1.453 & $0.633-3.067$ & 0.416 \\
$\begin{array}{l}\text { Age (years) } \\
\geq 60 \text { vs }<60\end{array}$ & 0.621 & $0.37-1.113$ & 0.133 \\
$\begin{array}{l}\text { Tumor size (cm) } \\
\geq 5 \text { vs }<5\end{array}$ & 1.992 & $1.133-3.503$ & 0.017 \\
$\begin{array}{l}\text { Vascular invasion } \\
\text { Positive vs Negative }\end{array}$ & 3.577 & $1.866-6.855$ & $<0.001$ \\
$\begin{array}{l}\text { Pathological stage } \\
\text { III-IV vs I-II }\end{array}$ & 1.257 & $0.739-2.204$ & 0.388 \\
$\begin{array}{l}\text { T stage } \\
\text { T2-4 vs T1 }\end{array}$ & 2.247 & $0.894-5.649$ & 0.085 \\
$\begin{array}{l}\text { TNM stage } \\
\text { III-IV vs I-II }\end{array}$ & 2.871 & $1.648-5.003$ & $<0.001$ \\
$\begin{array}{l}\text { MMP28 expression } \\
\text { High vs Low }\end{array}$ & 2.778 & $1.505-5.129$ & $<0.001$ \\
\hline
\end{tabular}

Abbreviations: $\mathrm{HR}=$ hazard ratio; $95 \% \mathrm{CI}=95 \%$ confidence interval; $P<0.05$ is considered to have statistical significance.

\section{Predictive nomogram for survival of patients with HCC}

According to all these independent prognostic factors selected above, we constructed prognostic nomogram for predicting 5-year survival rate in patients with HCC. Each independent prognostic factor had a risk score and the total point was raised by adding the score of MMP28 expression ( 0 for "Low", 91 for "High"), the TNM stage (0 for "I", 20 for "II", 46 for "III", 100 for "IV"). Higher total point reflected a lower survival rate (Fig. 3a). Then the patients were divided into three subgroup based on the total risk score: subgroups I, low risk score (< $25 \%)$; subgroup II, medium risk score (25\%-75\%); and subgroup III, high risk score (> 75\%). Kaplan-Meier curves showed that scoring with MMP28-based nomogram remarkably distinguished the risk of postoperative survival of HCC patients $(P<0.001)$ (Fig. 3b).

\section{TNM staging prognostic model for HCC patients is improved by the combination of MMP28 expression}

To establish a more reasonable model to predict the outcomes of HCC patients, TNM staging system was combined with MMP28 expression. ROC curve analysis was employed to show the prognostic sensitive and specificity of MMP28 expression, TNM staging system and the combined system. Our results indicated that the combined system revealed a significantly better prognostic value (AUC $[95 \% \mathrm{CI}]$, 0.734 [0.622-0.845]) than MMP28 expression alone (AUC [95\% CI], 0.663 [0.541-0.784]) or TNM staging system alone (AUC [95\% CI], 0.681 [0.564-0.798]) (Fig. $3 \mathrm{c})$. In addition, the Akaike information criterion (AIC) for the combination of TNM stage and MMP28 was 394.87, lower than that for TNM stage (404.27), whereas the Harrell's concordance index (C-index) of combination model was increased to 0.677 compared with that of TNM stage (0.640) alone (Fig. 3d). For internal validation, the calibration curves revealed that the nomogram predicting 5-year overall survival rate performed well with the ideal prediction model (Fig. 3e). These date demonstrated that the novel system could generate a more accurate predictive model for the prognosis of HCC patients.

\section{MMP28 promotes EMT, migration and invasion of HCC cells}

We next evaluated effect of MMP28 on abilities of both migration and invasion of HCC cells in vitro. Our data revealed that MMP28 was differently expressed in different HCC cell lines (Supplementary Fig S1). BEL-7402 cells with low basal expression were transfected with MMP28 overexpression plasmids, 
and Huh7 cells with high basal expression were transfected with MMP28 small-interfering RNA (siRNA). The overexpressing and interfering efficiency was determined by WB and qPCR (Fig. 4a). The transwell migration and invasion assays were employed to confirm the aggressive ability of the HCC cells, and the results revealed that both the migrated and invasive cells were significantly increased in MMP28overexpressing group, and significantly decreased in
MMP28-knock-down group, compared with control ones (Fig. 4b, c). Consistent with the results, we found that mice with high level MMP28 expression appeared more frequent metastasis (Fig. 4d), and MMP28 upregulation significantly decreased the survival of tumor-bearing mice $(P=0.039)$ (Fig. $4 \mathrm{e})$. These data indicated that MMP28 could promote migration and invasion in vivo and in vitro.

a
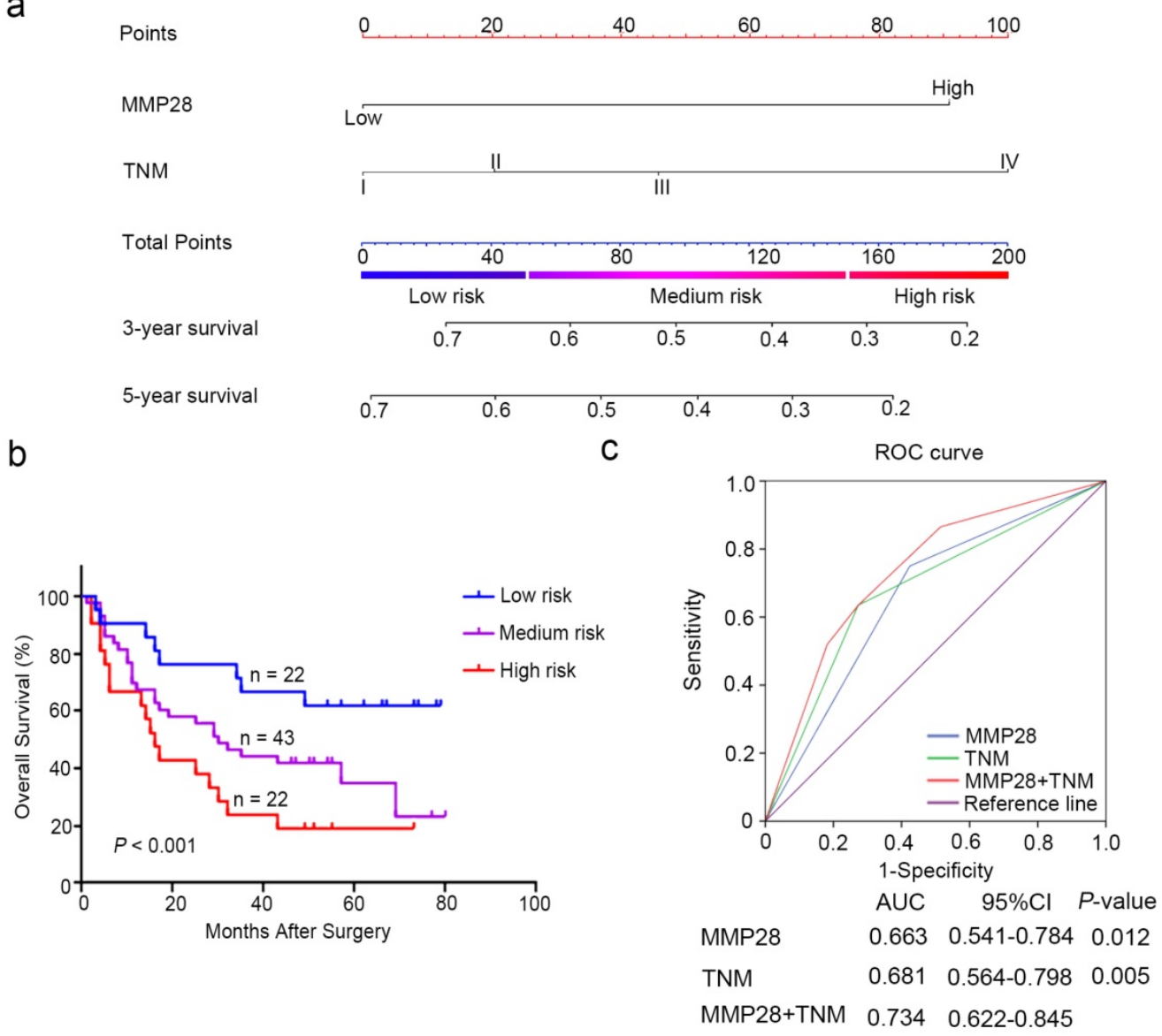

d

\begin{tabular}{|ccc|}
\hline \multicolumn{3}{|c|}{$\begin{array}{c}\text { Predictive Accuracies of } \\
\text { Prognositic Models }\end{array}$} \\
\hline Models & AIC & C-index \\
\hline MMP28 & 405.873 & 0.63125 \\
\hline TNM & 404.265 & 0.63952 \\
\hline MMP28+TNM & 394.871 & 0.67671 \\
\hline AIC: & akaike information criterion \\
\hline C-index: & Harrell's concordance index \\
\hline
\end{tabular}

e

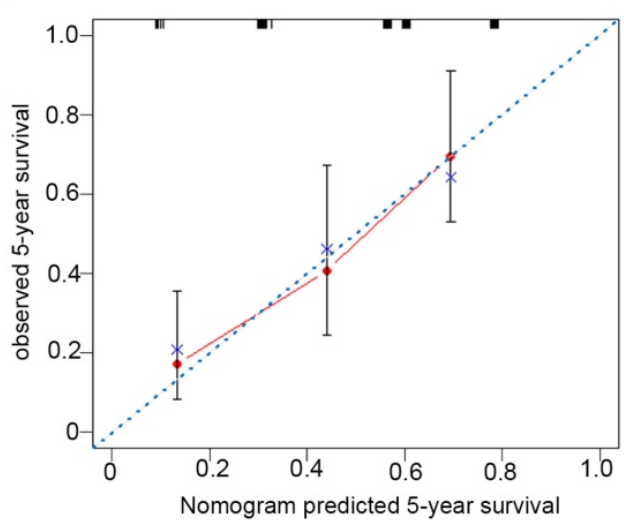

Figure 3. Combination of MMP28 expression with TNM stage established a better predictive model to predict the survival rate of HCC patients. (a) Nomogram integrated MMP28 expression and TNM stage. (b) Kaplan-Meier survival analysis of overall survival according to the risk score calculated by the nomogram. (c) ROC curve analysis of the specificity and sensitivity of the predictive value of the MMP28 model, TNM stage model and the combined model. (d) AIC and Harrell's C-index analysis of the predictive accuracies of the three models. (e) The calibration plots for predicted nomogram and actual 5-year overall survival. The dash line was regarded as an ideal model. 
a

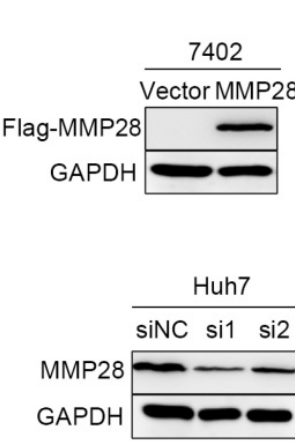

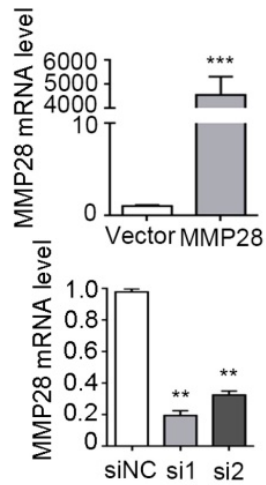

b

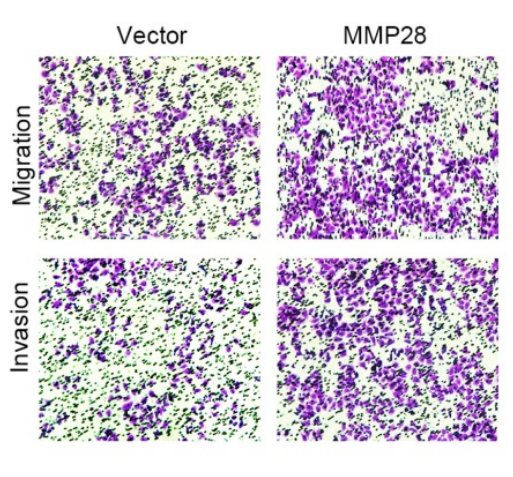

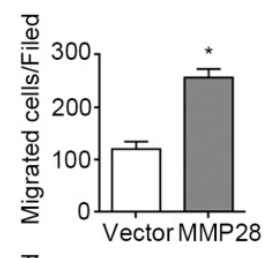

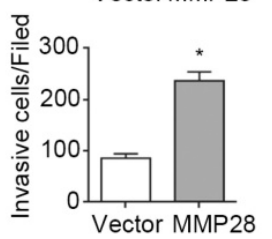

C

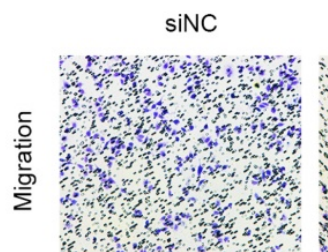

si1
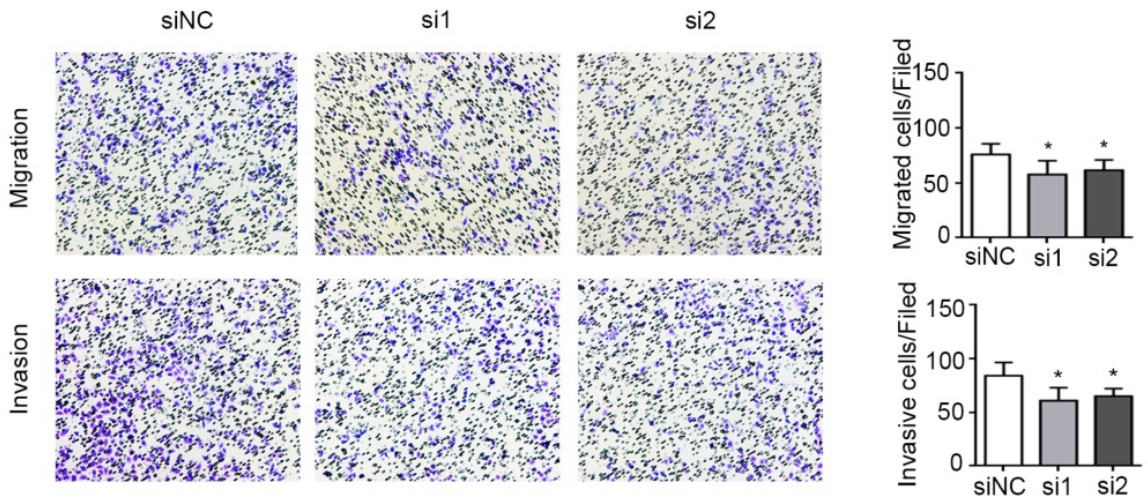

d

e
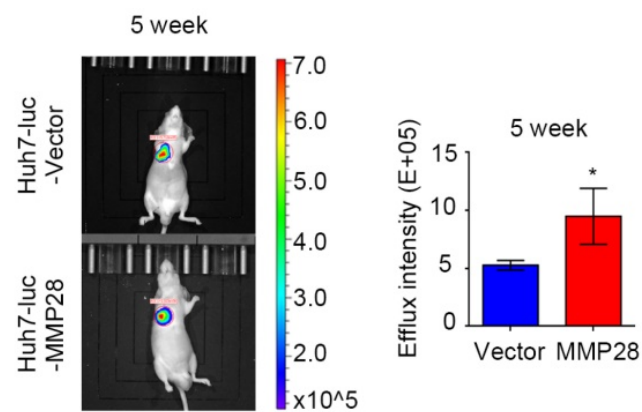

Overall survival of tumor-bearing mouse

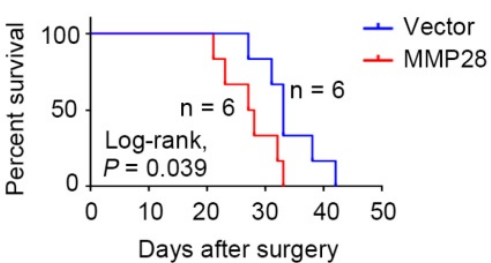

Figure 4. MMP28 promoted migratory and invasive abilities of HCC cells in vivo and in vitro. (a) The overexpression and knockdown efficiencies of MMP28 in cells were detected by western blot and qPCR. (b) Transwell assays were performed to determine the influence of MMP28 overexpressing on the migratory and invasive abilities in 7402 cells. (c) Transwell assays were performed to determine the influence of MMP28 silencing on the migratory and invasive abilities in Huh7 cells. (d) In vivo effect of MMP28 in lung metastasis model 5 weeks after Huh7-luc cells injected. Representative images of metastasis models were presented on the left, and statistical data was presented on the right. (e) Overall survival of grouped mice. $* P<0.05$; ** $P<0.01$; *** $P<0.001$. Data were represented as Means \pm SD.

Since EMT is considered to be the first step in metastasis, the involvement of molecules in this process varies among different cancer types[33]. Thus we determined the effect of MMP28 on the EMT-related hallmarks. Compared with the control, we found the EMT-inducing transcription factors ZEB-1, ZEB-2, Snail and mesenchymal marker $\mathrm{N}$-cadherin were significantly upregulated in MMP28-overexpressing cells and downregulated in MMP28-knock-down cells respectively, while the epithelial marker E-cadherin was repressed in
MMP28-overexpressing group and elevated in MMP28-knock-down group respectively (Fig. 5a). WB analysis showed the corresponding effects on the protein level of these factors (Fig. 5b, c). However, in parallel IHC analysis, it suggested the expression level of MMP28 was positively correlated only with ZEB-1 and ZEB-2 ( $\mathrm{R}=0.297, P=0.011$ for ZEB- 1 and $\mathrm{R}$ $=0.331, P=0.004$ for ZEB-2) (Fig. 5e, f), which was similar to the data from TCGA dataset $(\mathrm{R}=0.182, P<$ 0.001 for ZEB-1 and $\mathrm{R}=0.223, P<0.001$ for ZEB-2) (Fig. 5d). 
a

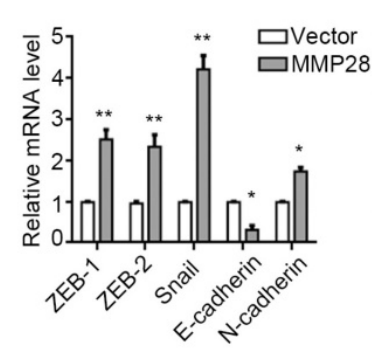

b

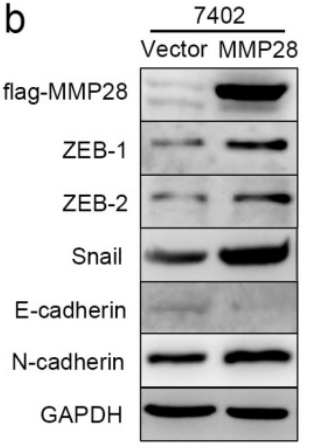

C

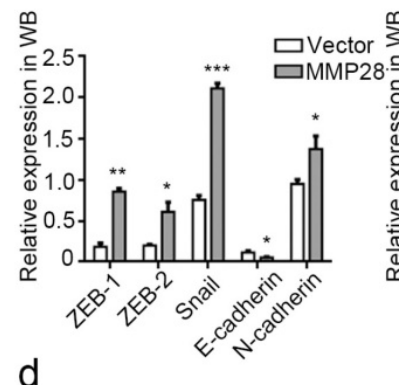

d

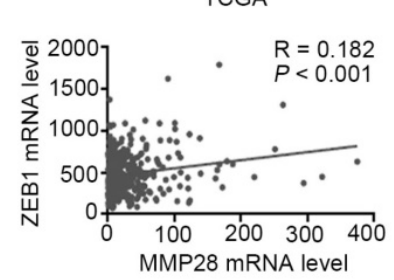

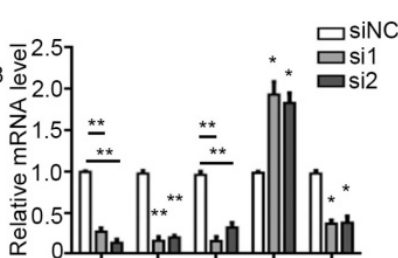
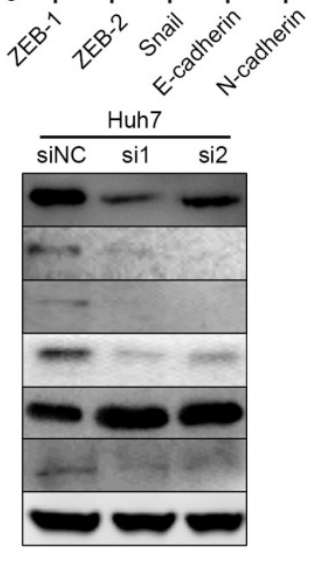

e
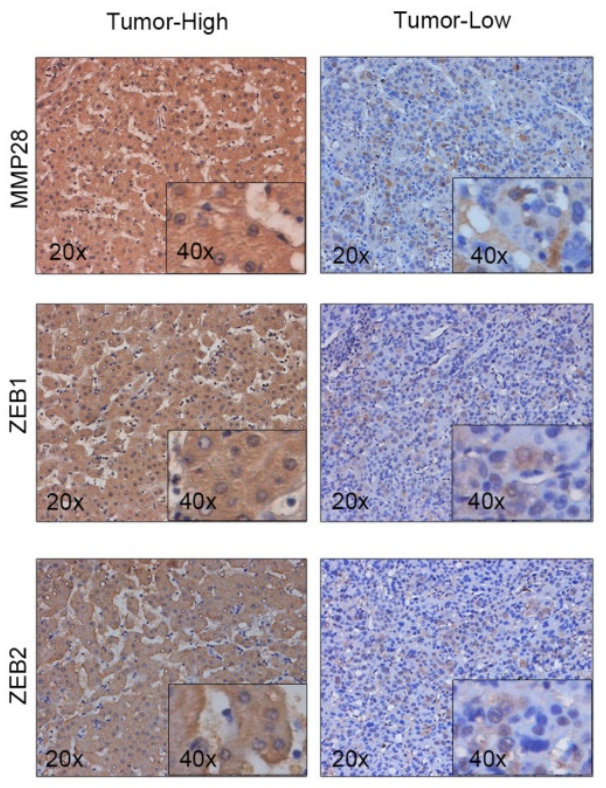

\section{f}
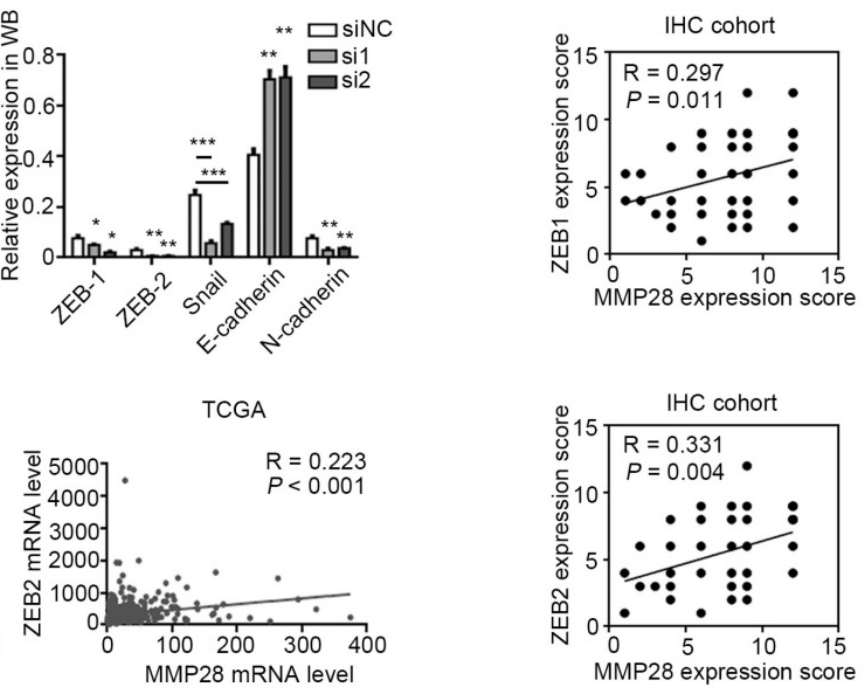

Figure 5. MMP28 promoted EMT of HCC cells. (a-c) The effect of MMP28 overexpression and knockdown on the expression of EMT-related factors in 7402 and Huh7 cells were determined by real-time PCR (a) and western blot (b, c). (d) The correlations of MMP28 with ZEB-1 and ZEB-2 in TCGA dataset. (e) IHC assays of MMP28, ZEB-1 and ZEB-2. Representative IHC images were shown. (f) The correlations of MMP28 with ZEB-1 and ZEB-2 in IHC cohort. $* P<0.05 ; * * P<0.01$; $* * * P<0.001$. Data were represented as Means \pm SD

\section{MMP28 promotes EMT via activating Notch3 signaling}

To further investigate how MMP28 upregulates the aggressive abilities of HCC cells, the genes strongly correlated with MMP28 from TCGA database were all taken into account (Pearson $|R| \geq 0.3$ and Spearman $|R| \geq 0.3$ ). We sorted out 837 genes that strongly correlated with MMP28 (Supplementary Fig S2). A considerable amount of the genes were significantly correlated with environmental information processing in functional enrichments of the KEGG pathway (Supplementary Fig S3 and Supplementary Table S1). We selected several feasible signaling pathways that are relatively significant to search the molecules involved, including Notch signaling, AKT signaling, and PKG signaling, and performed western blot analysis to examine the expression of relevant molecules. Phosphorylated- Ser239VASP was used to reflect the activity of PKG-I[34]. Our results showed that among these signals, NICD3 was elevated in MMP28-overexpressing cells (Fig. 6a, b). And in MMP28-knockdowned cells, NICD3 was also significantly downregulated (Fig. 6c). Analysis on TCGA dataset also revealed the positive correlation between MMP28 and Notch3 $(\mathrm{R}=0.556, P<0.001)$ (Fig. 6d), which was confirmed in qPCR analysis $(R=$ 
0.362, $P=0.004)$ and IHC analysis $(\mathrm{R}=0.294, P=0.012)$ on our HCC samples (Fig. 6e-g). And the positive staining area of NICD3 in cytoplasm was increased in MMP28-high tumor tissues (Fig. 6f). In conclusion, MMP28 is associated with Notch3 activation.

\section{The effects of MMP28 on HCC progression depend on Notch3 activation}

To further examine whether Notch3 signaling is involved in the functions of MMP28, we generated a stable Huh7 cell line with Notch3 knockout (N3-KO) using CRISPR/Cas9 system. The knockout efficacy was determined by western blot (Fig. 7a). In N3-KO cells, the expression of ZEB-1 and ZEB-2 was downregulated (Fig. 7a). And transwell assays showed that N3-KO cells exhibited less migratory and invasive potentials than parental cells (Fig. 7b). Moreover, neither overexpression nor knockdown of MMP28 in N3-KO cells conferred significant effects on migration or invasion of N3-KO cells (Fig. 7d, f). ZEBs levels were also not changed at the same time (Fig. 7c, e). Collectively, these data indicated that Notch3 is required for the promotion of MMP28 on EMT, migration and invasion of HCC cells.

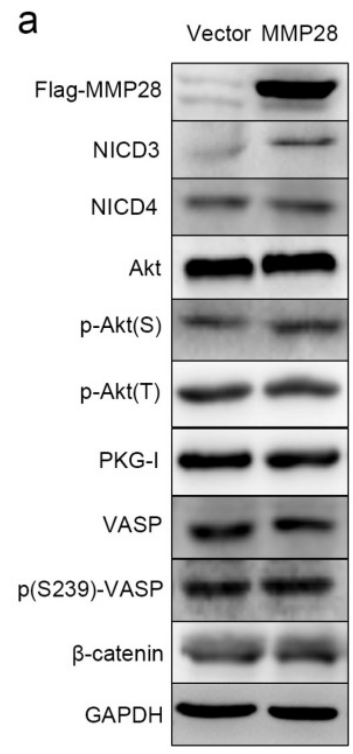

d
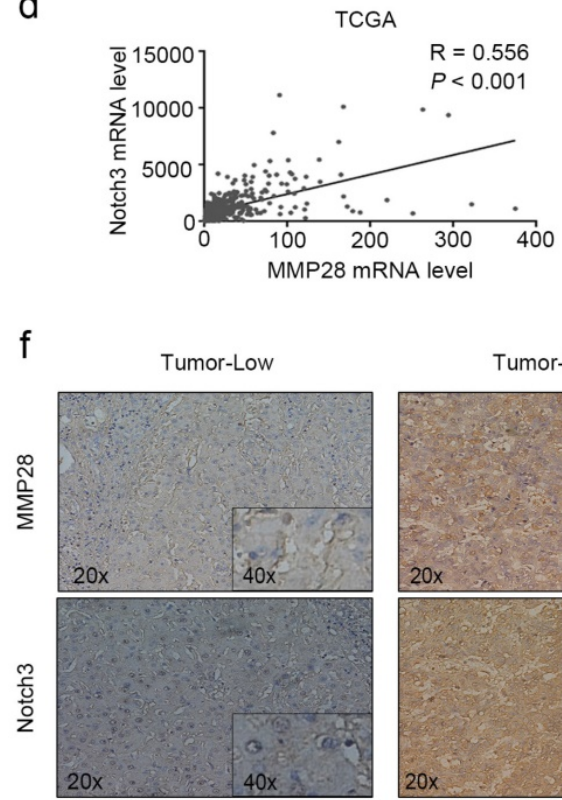

b
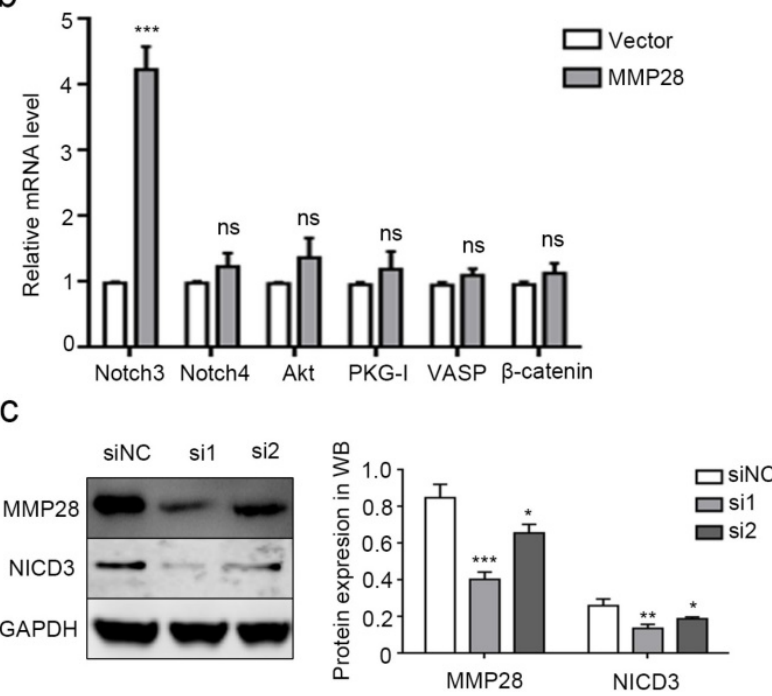

e

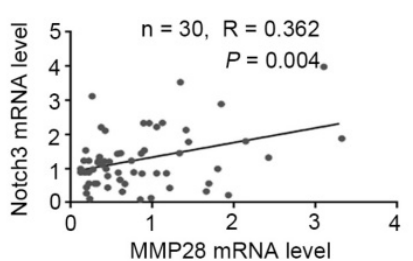

g
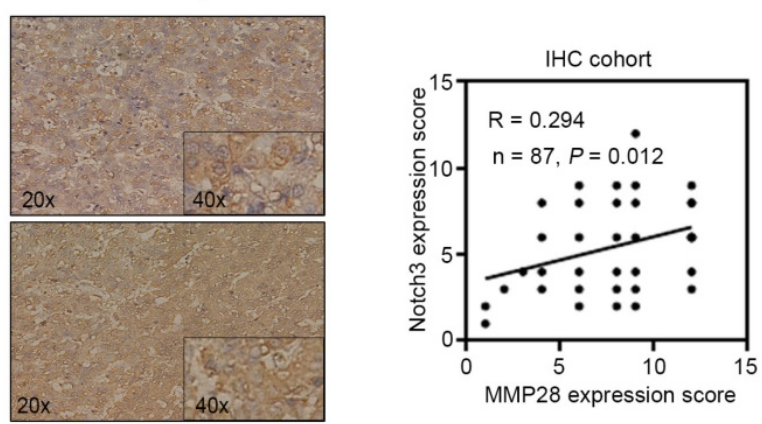

Figure 6. MMP28 promoted EMT, migration and invasion of HCC cells via activating Notch3 signals. (a) Levels of NICD3, NICD4, Akt, p-Akt(S), P-Akt(T), PKG-I, VASP, $\mathrm{p}(\mathrm{S} 239)$-VASP and $\beta$-catenin in MMP28-overexpressing 7402 cells were determined by western blot. (b) The mRNA levels of Notch3, Notch4, Akt, PKG1, VASP and $\beta$-catenin in MMP28-overexpressing 7402 cells was determined by real-time PCR. (c) The expression of MMP28 and NICD3 in MMP28-knockdowned Huh7 cells were determined by western blot. (d) Expression correlation between MMP28 and Notch3 from TCGA dataset. (e) Correlation of MMP28 mRNA levels with Notch3 in HCC tissues was determined by real-time PCR. (f-g) Correlation of MMP28 mRNA levels with Notch3 in IHC cohort was determined by IHC analysis. Representative images of MMP28 and Notch3 in HCC tissues were shown. $* P<0.05$; **P $<0.01$; ***P $<0.001$. Data are represented as Means \pm SD. 
a

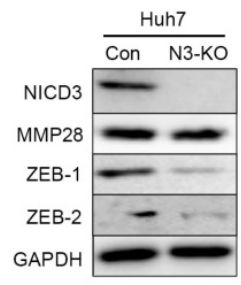

C

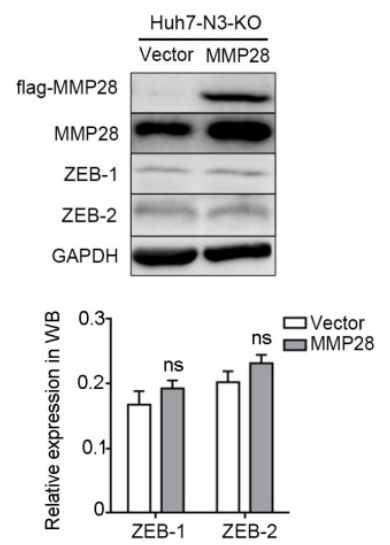

b
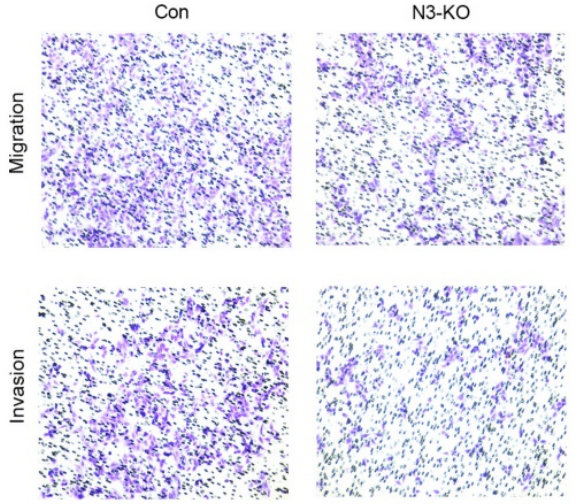

d
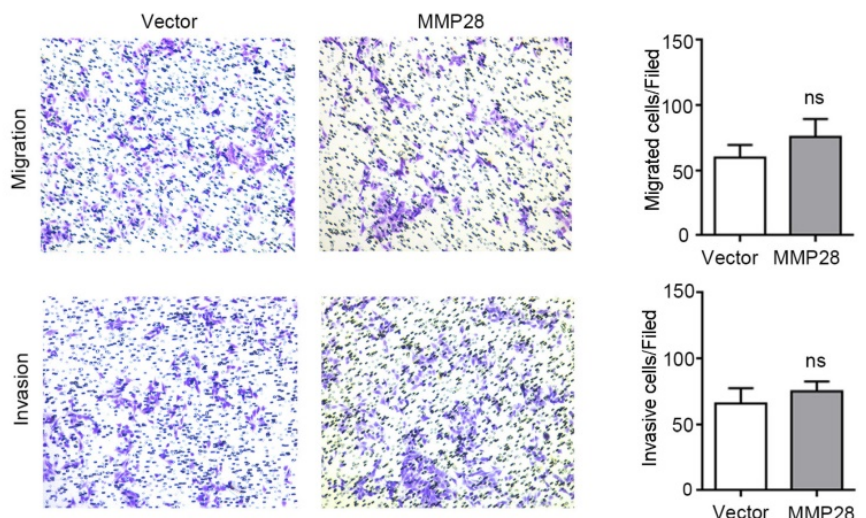
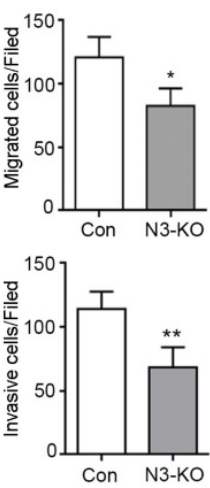

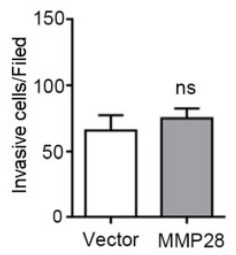

e
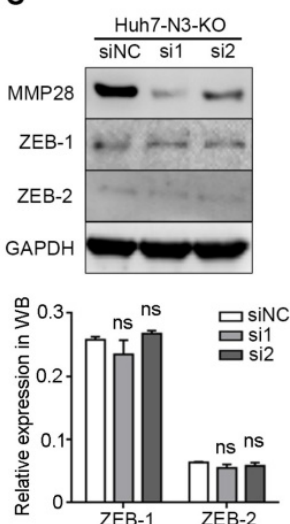

f
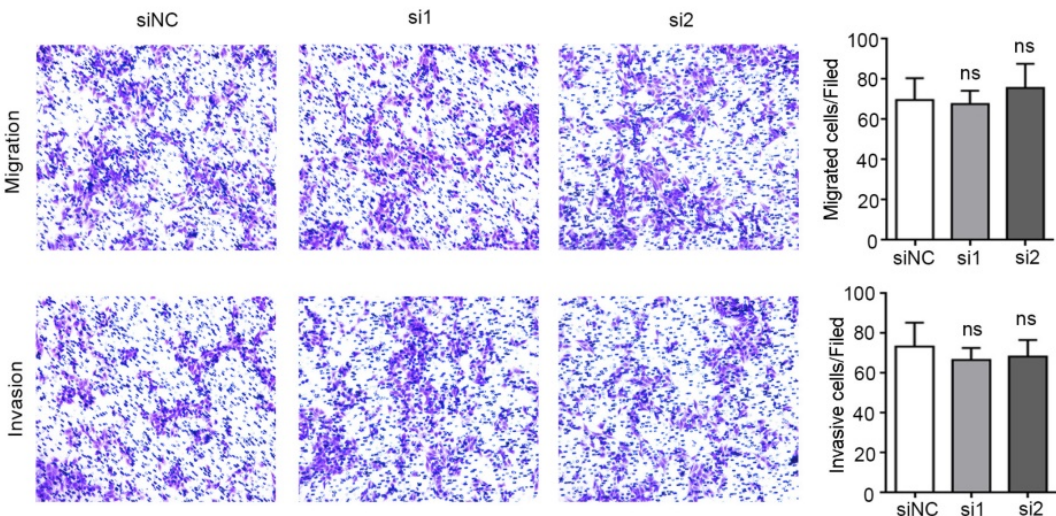

Figure 7. Notch3 knockout dispelled the metastasis-promoting ability of MMP28. (a) Levels of NICD3, MMP28, ZEB-1 and ZEB-2 in Notch3-knockout Huh7 stable cells (N3-KO) were determined by western blot. (b) Transwell assays were performed to determine the influence of Notch3 knockout on the migratory and invasive abilities of Huh7 stable cells. (c) The expression of ZEB-1 and ZEB-2 in N3-KO cells transfected with MMP28 was determined by western blot. (d) Transwell assays were performed to determine the influence of MMP28 overexpressing on the migratory and invasive abilities in N3-KO cells. (e) The expression of ZEB-1 and ZEB-2 in N3-KO cells transfected with MMP28 siRNA was determined by western blot. ( $f$ ) Transwell assays were performed to determine the influence of MMP28 silencing on the migratory and invasive abilities in N3-KO cells. $* P<0.05 ; * * P<0.01$. Data are represented as Means \pm SD.

\section{Discussion}

In this study, our data revealed that MMP28 is upregulated in HCC tumor tissues, which is associated with elevated vascular invasion, higher TNM stage and worse overall survival. Upregulated MMP28 was also identified as an independent prognosis factor to predict the poor outcomes of patients with HCC. Furthermore, since TNM staging system does not take the heterogeneity of HCC into consideration and thus is not sufficiently accuracy, we developed a novel model combining MMP28 expression and TNM staging system to improve the accuracy of the predictions. All these results indicate that MMP28 could be used as a potential biomarker. 
Pathologically, the expression and the activation of MMPs are abnormal in human cancers, which are involved in several critical processes including tumor proliferation, differentiation, migration, invasion, angiogenesis, and apoptosis [35]. MMPs are often aberrantly upregulated in cancers, which leads to unsatisfied results. For example, MMP13 secreted from fibroblasts promotes tumor angiogenesis [36], and MMP2 and MMP9 degrades ECM components to promote metastasis [37]. MMP28 is also benefit for tumor progression. MMP28 is initially is found in the basal keratinocytes and fetal tissues, and functions in tissue homeostasis and development [38]. Further evidence suggests that MMP28 expression is induced by tumor necrosis factor a (TNF-a), so as to accelerate wound healing [15]. Similar with its epithelial repair ability in normal tissues, MMP28 promotes proliferation of tumor cells in oral squamous cell carcinomas (OSCCs) and esophageal carcinomas [39]. And a recent research demonstrated that high levels of MMP28 predict unsatisfactory prognosis in gastric carcinoma, and are correlate with the gastric tumor invasion and metastasis [40]. Here, our data proved that MMP28 is also upregulated in HCC, and promote metastasis.

In epithelial cells, upregulated MMP28 induces EMT via activating TGF- $\beta$ and decreasing a major mediator of cell-cell adhesion, E-cadherin [41]. The transient activity of MMP28 in A549 lung adenocarcinoma cells causes TGF- $\beta$-dependent EMT as well, which leads to the atypical hyperplasia and invasive tendency of cancer cells [42]. Meanwhile, one of the substrates of MMP28, casein, has been identified as a novel inhibitor of tumor progression [43, 44]. Additionally, several TIMPs that inhibit the activity of MMP28 suppress the progression of breast carcinoma [45], and colorectal cancer [46], and have been used in treating cancers [47]. Considering the clinicopathologic roles of MMP28 in HCC, targeting MMP28 might also be a promising strategy in clinical practice for HCC patients.

EMT is a histopathological process that occurs in abnormal cells, which destroys the links between tumor cells and the ECM, and enhances the ability of aggressiveness for tumor cells. Notch signaling pathway, that regulates local cell-cell communication and cell differentiation [48], is related to the EMT process [49]. Notch3 promoted EMT and tumor initiation in human squamous cell carcinoma [50], and the mutations on Notch3 disrupted the expansion of EMT-related cells in non-small cell lung cancer [51]. Notch3 also induces the formation of metastasis lesions in the pancreatic ductal adenocarcinoma cells via MMP2 and MMP9 [52]. Although the overexpression of Notch3 has been reported in HCC
[27], little is known about the functions of Notch3 in HCC. Here, we proved that activated Notch3 could regulate the expression of ZEB homologues in HCC tissues, thus to promote EMT. And Notch3 was involved in MMP28-triggered tumor cell infiltration. However, although in vitro results showed that the upregulation of MMP28 led to the expression changes of Snail, E-cadherin and N-cadherin, we failed to find similar results in IHC data of HCC patients. These data suggested that despite the direct promotion of MMP28 and Notch3 on migration and invasion of HCC tumor cells, the effects of MMP28 and Notch3 on tumors might be changed by some other factors in microenvironment, which requires further investigation.

In conclusion, this study explored the expression pattern of MMP28 in HCC, and identified the upregulation of MMP28 as an independent prognostic factor in HCC patients. Meanwhile, a more sensitive predict model for HCC patients combining MMP28 expression with TNM system was established. Moreover, our study revealed the effects of MMP28 on metastasis depend on Notch3 signals. Future studies are required to investigate the precise molecular mechanisms underlying the association between activated Notch3 and MMP28.

\section{Supplementary Material}

Supplementary figures and table.

http://www.ijbs.com/v15p0812s1.pdf

\section{Acknowledgements}

This work was supported by grants from the National Natural Science Foundation For Young Scientists of China (31300676), Foundation of Science Technology Department of Zhejiang Province (2013C3 3098), Foundation of Wenzhou Science \&Technology Bureau (Y20140110).

\section{Competing Interests}

The authors have declared that no competing interest exists.

\section{References}

1. Forner A, Bruix J. Hepatocellular carcinoma - Authors' reply. Lancet. 2012; 380: 470-1.

2. Gong Y, Wu X, Wang T, Zhao J, Liu X, Yao Z, et al. Targeting PEPT1: a novel strategy to improve the antitumor efficacy of Doxorubicin in human Hepatocellular carcinoma therapy. Oncotarget. 2017; 8: 40454-68.

3. Taylor EJ, Jones RL, Guthrie JA, Rowe IA. Modelling the benefits and harms of surveillance for hepatocellular carcinoma: information to support informed choices. Hepatology. 2017; 66: 1546.

4. Malaguarnera G, Giordano M, Paladina I, Berretta M, Cappellani A, Malaguarnera M. Serum markers of hepatocellular carcinoma. Digestive diseases and sciences. 2010; 55: 2744-55.

5. Zhang X, Huang S, Guo J, Zhou L, You L, Zhang T, et al. Insights into the distinct roles of MMP-11 in tumor biology and future therapeutics (Review). International Journal of Oncology. 2016; 48.

6. Boon L, Ugarteberzal E, Vandooren J, Opdenakker G. Glycosylation of matrix metalloproteases and tissue inhibitors: present state, challenges and opportunities. Biochemical Journal. 2016; 473: 1471-82. 
7. Pardo A, Selman M. Role of matrix metaloproteases in idiopathic pulmonary fibrosis. Fibrogenesis Tissue Repair. 2012; 5: 1-5.

8. Hu J, Steen PEVD, Sang QXA, Opdenakker G. Matrix metalloproteinase inhibitors as therapy for inflammatory and vascular diseases. Nature Reviews Drug Discovery. 2007; 6: 480.

9. Milner JM, Cawston TE. Matrix metalloproteinase knockout studies and the potential use of matrix metalloproteinase inhibitors in the rheumatic diseases. Current Drug Targets-Inflammation \& Allergy. 2005; 4: 363-75.

10. Kessenbrock K, Plaks V, Werb Z. Matrix metalloproteinases: regulators of the tumor microenvironment. Cell. 2010; 141: 52-67.

11. Wieczorek E, Jablonska E, Wasowicz W, Reszka E. Matrix metalloproteinases and genetic mouse models in cancer research: a mini-review. Tumour Biology the Journal of the International Society for Oncodevelopmental Biology \& Medicine. 2015; 36: 163-75

12. Wu J, Hao ZW, Zhao YX, Yang XM, Tang H, Zhang X, et al. Full-length soluble CD147 promotes MMP-2 expression and is a potential serological marker in detection of hepatocellular carcinoma. Journal of Translational Medicine. 2014; 12: 190 .

13. Illman SA, Lohi J, Keski-Oja J. Epilysin (MMP-28)--structure, expression and potential functions. Experimental dermatology. 2008; 17: 897-907.

14. Marchenko GN, Strongin AY. MMP-28, a new human matrix metalloproteinase with an unusual cysteine-switch sequence is widely expressed in tumors. Gene. 2001; 265: 87-93.

15. Saarialho-Kere U, Kerkelä E, Jahkola T, Suomela S, Keski-Oja J, Lohi J. Epilysin (MMP-28) expression is associated with cell proliferation during epithelial repair. Journal of Investigative Dermatology. 2002; 119: 14-21.

16. Jian P, Yanfang T, Zhuan Z, Jian W, Xueming Z, Jian N. MMP28 (epilysin) as a novel promoter of invasion and metastasis in gastric cancer. Bmc Cancer. 2011; 11: $1-8$.

17. Overall CM, Tam EM, Kappelhoff R, Connor A, Ewart T, Morrison CJ, et al. Protease degradomics: mass spectrometry discovery of protease substrates and the CLIP-CHIP, a dedicated DNA microarray of all human proteases and inhibitors. Biological Chemistry. 2004; 385: 493-504.

18. Capaccione KM, Pine SR. The Notch signaling pathway as a mediator of tumor survival. Carcinogenesis. 2013; 34: 1420-30

19. Andersson ER, Lendahl U. Therapeutic modulation of Notch signalling $\mid[$ mdash $] \mid$ are we there yet? Nature Reviews Drug Discovery. 2014; 13: $357-78$

20. Castel D, Mourikis P, Bartels SJJ, Brinkman AB, Tajbakhsh S, Stunnenberg HG. Dynamic binding of RBPJ is determined by Notch signaling status. Genes \& Development. 2013; 27: 1059-71.

21. Nijjar SS, Wallace L, Crosby HA, Hubscher SG, Strain AJ. Altered Notch Ligand Expression in Human Liver Disease : Further Evidence for a Role of the Notch Signaling Pathway in Hepatic Neovascularization and Biliary Ductular Defects. American Journal of Pathology. 2002; 160: 1695-703.

22. Ning L, Wentworth L, Chen H, Weber SM. Down-regulation of Notch1 signaling inhibits tumor growth in human hepatocellular carcinoma. American Journal of Translational Research. 2009; 1: 358.

23. Villanueva A, Alsinet C, Yanger K, Hoshida Y, Zong Y, Toffanin S, et al. Notch signaling is activated in human hepatocellular carcinoma and induces tumor formation in mice. Gastroenterology. 2012; 143: 1660.

24. Roy M, Pear WS, Aster JC. The multifaceted role of Notch in cancer. Current Opinion in Genetics \& Development. 2007; 17: 52-9.

25. Qi R, An H, Yu Y, Zhang M, Liu S, Xu H, et al. Notch1 signaling inhibits growth of human hepatocellular carcinoma through induction of cell cycle arrest and apoptosis. Cancer research. 2003; 63: 8323.

26. Hu L, Xue F, Shao M, Deng A, Wei G. Aberrant expression of Notch3 predicts poor survival for hepatocellular carcinomas. Bioscience Trends. 2013; 7: 152.

27. Gramantieri L, Giovannini C, Lanzi A, Chieco P, Ravaioli M, Venturi A, et al. Aberrant Notch3 and Notch4 expression in human hepatocellular carcinoma. Liver International. 2007; 27: 997-1007.

28. Gao J, Song Z, Chen Y, Xia L, Wang J, Fan R, et al. Deregulated expression of Notch receptors in human hepatocellular carcinoma. Dig Liver Dis. 2008; 40: $114-21$.

29. Lim HY, Sohn I, Deng S, Lee J, Jung SH, Mao M, et al. Prediction of disease-free survival in hepatocellular carcinoma by gene expression profiling. Annals of surgical oncology. 2013; 20: 3747-53.

30. Tung EK, Mak CK, Fatima S, Lo RC, Zhao $H$, Zhang $C$, et al. Clinicopathological and prognostic significance of serum and tissue Dickkopf-1 levels in human hepatocellular carcinoma. Liver International. 2011; 31: 1494-504

31. Hoon KJ, Hwa SB, Hyun-Sung L, Sang-Bae K, Eun YJ, Yun-Yong P, et al. Genomic Predictors for Recurrence Patterns of Hepatocellular Carcinoma: Model Derivation and Validation. PLoS medicine. 2014; 11: e1001770.

32. Tomczak K, Czerwińska P, Wiznerowicz M. The Cancer Genome Atlas (TCGA): an immeasurable source of knowledge. Contemporary Oncology. 2015; 19: 68-77.

33. Kalluri R, Weinberg RA. The basics of epithelial-mesenchymal transition. Journal of Clinical Investigation. 2009; 119: 1420-8.

34. Wang S, Lincoln TM, Murphyullrich JE. Glucose downregulation of PKG-I protein mediates increased thrombospondin1-dependent TGF-\{beta\} activity in vascular smooth muscle cells. American Journal of Physiology Cell Physiology. 2010; 298: C1188.

35. Masson V. [Roles of serine proteases and matrix metalloproteinases in tumor invasion and angiogenesis]. Bull Mem Acad R Med Belg. 2006; 161: 320-6.
36. Kudo $\mathrm{Y}$, Iizuka S, Yoshida $\mathrm{M}$, Tsunematsu $\mathrm{T}$, Kondo $\mathrm{T}$, Subarnbhesaj A, et al. Matrix Metalloproteinase-13 (MMP-13) Directly and Indirectly Promotes Tumor Angiogenesis. Journal of Biological Chemistry. 2012; 287: 38716-28.

37. Shipley JM, Doyle GA, Fliszar CJ, Ye Q-Z, Johnson LL, Shapiro SD, et al. The Structural Basis for the Elastolytic Activity of the $92-\mathrm{kDa}$ and $72-\mathrm{kDa}$ Gelatinases ROLE OF THE FIBRONECTIN TYPE II-LIKE REPEATS. Journal of Biological Chemistry. 1996; 271: 4335-41.

38. Lohi J, Wilson CL, Roby JD, Parks WC. Epilysin, a novel human matrix metalloproteinase (MMP-28) expressed in testis and keratinocytes and in response to injury. Journal of Biological Chemistry. 2001; 276: 10134-44.

39. Lin MH, Liu SY, Su HJ, Liu YC. Functional role of matrix metalloproteinase-28 in the oral squamous cell carcinoma. Oral Oncology. 2006; 42: 907-13.

40. Jian P, Yanfang T, Zhuan Z, Jian W, Xueming Z, Jian N. MMP28 (epilysin) as a novel promoter of invasion and metastasis in gastric cancer. BMC cancer. 2011; 11: 200.

41. Illman SA, Lehti K, Keski-Oja J, Lohi J. Epilysin (MMP-28) induces TGF-beta mediated epithelial to mesenchymal transition in lung carcinoma cells. Journal of Cell Science. 2006; 119: 3856-65.

42. Illman SA, Lehti K, Keskioja J, Lohi J. Epilysin (MMP-28) induces TGF-beta mediated epithelial to mesenchymal transition in lung carcinoma cells. Journal of Cell Science. 2006; 119: 3856.

43. Bonuccelli G, Castello-Cros R, Capozza F, Martinez-Outschoorn UE, Lin Z, Tsirigos A, et al. The milk protein a-casein functions as a tumor suppressor via activation of STAT1 signaling, effectively preventing breast cancer tumor growth and metastasis. Cell cycle. 2012; 11: 3972-82.

44. Le Parc A, Houeto EH, Pigat N, Chat S, Leonil J, Chanat E. The membrane-associated form of as1-casein interacts with cholesterol-rich detergent-resistant microdomains. PloS one. 2014; 9: e115903.

45. Ławicki S, Zajkowska M, Głażewska EK, Będkowska GE, Szmitkowski M. Plasma levels and diagnostic utility of VEGF, MMP-2 and TIMP-2 in the diagnostics of breast cancer patients. Biomarkers. 2017; 22: 157-64.

46. Lorenc Z, Waniczek D, Lorenc-Podgórska K, Krawczyk W, Domagała M, Majewski M, et al. Profile of Expression of Genes Encoding Matrix Metallopeptidase 9 (MMP9), Matrix Metallopeptidase 28 (MMP28) and TIMP Metallopeptidase Inhibitor 1 (TIMP1) in Colorectal Cancer: Assessment of the Role in Diagnosis and Prognostication. Medical Science Monitor. 2017; 23: 1305-11.

47. Folgueras AR, Pendás AM, Sánchez LM, Lópezotín C. Matrix metalloproteinases in cancer: from new functions to improved inhibition strategies. International Journal of Developmental Biology. 2004; 48: 411-24.

48. Artavanistsakonas S, Rand MD, Lake RJ. Notch signaling: cell fate control and signal integration in development. Science. 1999; 284: 770.

49. Illam SP, Narayanankutty A, Mathew SE, Valsalakumari R, Jacob RM, Raghavamenon AC. Epithelial Mesenchymal Transition in Cancer Progression: Preventive Phytochemicals. Recent Pat Anticancer Drug Discov. 2017; 12: 1-1

50. Natsuizaka M, Whelan KA, Kagawa S, Tanaka K, Giroux V, Chandramouleeswaran PM, et al. Interplay between Notch1 and Notch3 promotes EMT and tumor initiation in squamous cell carcinoma. Nature Communications. 2017; 8: 1758.

51. Li C, Song G, Zhang S, Wang E, Cui Z. Wnt3a increases the metastatic potential of non-small cell lung cancer cells in vitro in part via its upregulation of Notch3. Oncology Reports. 2015; 33: 1207

52. Zhou JX, Zhou L, Li QJ, Feng W, Wang PM, Li EF, et al. Association between high levels of Notch3 expression and high invasion and poor overall survival rates in pancreatic ductal adenocarcinoma. Oncology Reports. 2016; 36. 